\title{
ON THE INTERSECTION FORMS OF CLOSED 4-MANIFOLDS
}

\author{
Alberto Cavicchioli and Frigdricit Hegenbarth
}

Abstract

Given a closed 4-manifold $M$, let $M^{*}$ be the simply-connected 4-manifold obtained from $M$ by killing the fundamental group. We study the relation between the intersection forms $\lambda_{M}$ and $\lambda_{M} \cdot$. Finally some topological consequences and examples are described.

\section{Introduction.}

Let $M^{4}$ be a closed connected orientablc (PL) 4-manifold with fundamental group $\Pi_{1}$.

Denote by $\lambda_{M}$ the intersection form of $M$

$$
\lambda_{M}: F H_{2}(M) \times F H_{2}(M) \longrightarrow Z
$$

wherc $\mathrm{FH}_{2}(M)=\mathrm{H}_{2}(M ; Z) /$ torsion (see for example [5], [10]).

Let $M^{*}$ be the simply-connected closed 4-manifold obtained from $M$ by killing the fundamental group $\Pi_{1}$ (see [6]).

Our purpose is to study what relation links $\lambda_{M}$ to $\lambda_{M}$. . Then we obtain some topological consequences about $M^{*}$. Finally we give some examples which illustrate the results.

\section{Main results.}

Let $[\alpha]$ be a generator of $\Pi_{1}$. Since $M$ is orientable, we can extend $\alpha: S^{1} \longrightarrow M$ to an embedding $\psi: S^{1} \times D^{3} \longrightarrow M$.

Recall that therc are two ways to extend $\alpha$ since $\Pi_{1}(S O(3)) \cong Z_{2}$.

Work performed under the auspicies of the G.N.S.A.G.A. of the C.N.R. and financially supported by the Ministero della Ricerca Scientifica e Tecnologica of Italy within the project "Geometria Reale e Complessa" 
Denote by $M^{\prime}=M \backslash \psi\left(S^{1} \times \stackrel{\circ}{D^{3}}\right) \cup D^{2} \times S^{2}$ the closed 4-manifold obtained from $M$ by surgery on $\psi$.

Since $\Pi_{1}\left(M^{\prime}\right) \cong \Pi_{1}(M) /[\alpha]$, iterated surgeries on generators of $\Pi_{1}(M)$ give a simply-connected closed 4-manifold $M^{*}$.

Problem. Study the relations between $\lambda_{M}, \lambda_{M^{\prime}}$ and $\lambda_{M}, \lambda_{M^{*}}$ respect.ively.

First we have the following

Proposition 1. If $\Pi_{1}(M)$ has no elements of finite order, then $\lambda_{M^{*}}$ is isomorphic over the integers to $\lambda_{M}$.

The proof is given for example in [1] .

Therefore from now on we will consider manifolds with $\Pi_{1}(M)$ finite.

Proposition 2. If $\{\alpha\}$ has finite order, then

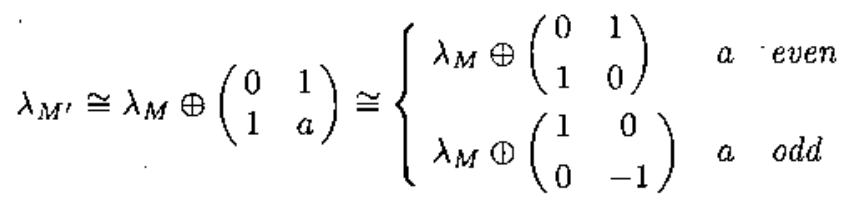

for some integer $a \in Z$.

In any case $\lambda_{M^{\prime}}$ is indefinite. For these forms there is the following well-known classification:

$$
\begin{aligned}
& \text { 1) } \lambda_{M^{\prime}} \text { everl } \Longrightarrow \quad \lambda_{M^{\prime}} \cong p E_{8} \oplus q\left(\begin{array}{ll}
0 & 1 \\
1 & 0
\end{array}\right) \\
& \text { 2) } \lambda_{M^{\prime}} \text { odd } \Longrightarrow \quad \lambda_{M^{\prime}} \cong p(1) \oplus q(-1)
\end{aligned}
$$

for some non negative integers $p, q \in Z$.

Furthermore, S. K. Donaldson (see [2]) proved the following

Theorem 3. Let $M^{4}$ be a closed connetied orientable 4-manifold with arbitrary fundamental group. If $\lambda_{M}$ is definite, then $\lambda_{M}$ is isomorphic over the integers to either $(1) \mathbb{D} \cdots \oplus(1)$ or $(-1) \oplus \cdots \oplus(-1)$.

The parity of $\lambda_{M}$ is related to the second Stiefel-Whitney class 
$w_{2}(M) \in H^{2}\left(M ; Z_{2}\right)$ as follows. Using the universal coefficient sequence $0 \longrightarrow \operatorname{Ext}\left(H_{1}(M) ; Z_{2}\right) \longrightarrow H^{2}\left(M ; Z_{2}\right) \longrightarrow \operatorname{Hom}\left(H_{2}(M) ; Z_{2}\right) \longrightarrow 0$, it is easily proved that $\lambda_{M}$ is even if and only if $w_{2}(M) \in \operatorname{Ext}\left(H_{1}(M) ; Z_{2}\right)$.

In particular, if $H_{1}(M)$ has no 2-torsion, then $\lambda_{M}$ is even if and only if $w_{2}(M)=0$.

Thus proposition 2 implies the following

Proposition 4. If $w_{2}(M) \neq 0$, then

$$
\lambda_{M} \cdot \cong \lambda_{M} \oplus p\left(\begin{array}{cc}
1 & 0 \\
0 & -1
\end{array}\right) \cong r(1) \oplus s(-1)
$$

for some non negative integers $p, r, s \in Z$.

Further, $M^{*}$ is homeomorphic to the connected sum $r\left(C P^{2}\right) \# s\left(-C P^{2}\right)$, being $C P^{2}$ the projective complex plane.

Now we can also apply theorem 2 of [2] to obtain the following consequence of proposition 2 .

Corollary 5. Let $M^{4}$ be a closed connected orientable spin 4-manifold with fundamental group $\Pi_{1}(M) \cong Z_{m}$.

If $\lambda_{M}$ has a positive part of rank 1 , then $M^{*}$ is homeomorphic to either $2\left(C P^{2}\right) \#(2-\sigma(M))\left(-C P^{2}\right)$ or $2\left(S^{2} \times S^{2}\right)$. $M$.

In the last case, $\lambda_{M} \cong\left(\begin{array}{ll}0 & 1 \\ 1 & 0\end{array}\right)$. Here $\sigma(M)$ denotes the signature of

Proof: By proposition 2, we have either $\lambda_{M} \cong \lambda_{M} \oplus\left(\begin{array}{ll}0 & 1 \\ 1 & 0\end{array}\right)$ or $\lambda_{M} \cong \lambda_{M} \oplus\left(\begin{array}{cc}1 & 0 \\ 0 & -1\end{array}\right)$, hence $\lambda_{M}$. has a positive part of rank 2 .

In the first case, $\lambda_{M}$. is even. Since $H_{1}\left(M^{*}\right) \cong 0$ has no 2 -torsion, theorem 2 of [2] implies that

$$
\lambda_{M} \cong\left(\begin{array}{ll}
0 & 1 \\
1 & 0
\end{array}\right) \oplus\left(\begin{array}{ll}
0 & 1 \\
1 & 0
\end{array}\right) \cong \lambda_{M} \oplus\left(\begin{array}{ll}
0 & 1 \\
1 & 0
\end{array}\right)
$$

hence $\lambda_{M} \cong\left(\begin{array}{ll}0 & 1 \\ 1 & 0\end{array}\right)$ (see $\left.[7] ;[9]\right)$ and $M^{*} \cong 2$

In the second case, $\lambda_{M} \cong 2(1) \oplus(2-\sigma(M))(-1)$, hence $M^{*} \underset{\mathrm{TOP}}{\cong} 2\left(C P^{2}\right) \#(2-\sigma(M))\left(-C P^{2}\right)$. 


\section{Examples.}

3.1) Let $K=\left\{z_{0}^{4}+z_{1}^{4}+z_{2}^{4}+z_{3}^{4}=0\right\} \subset C P^{3}$ be the Kummer surface and let $T: C P^{3} \longrightarrow C P^{3}$ be the fixed point free involution defined by

$$
T\left(z_{0}, z_{1}, z_{2}, z_{3}\right)=\left(\bar{z}_{1},-\bar{z}_{0}, \bar{z}_{3},-\bar{z}_{2}\right) \text {. }
$$

Since $T(K)=K$, we can consider the orbit space $M=K / T$, called the Habegger manifold (see [4]).

It is known that $\Pi_{1}(M) \cong Z_{2}$ and the intersection form

$$
\lambda_{M} \cong\left(-E_{8}\right) \oplus\left(\begin{array}{ll}
0 & 1 \\
1 & 0
\end{array}\right)
$$

is even with a positive part of rank 1.

Since $u_{2}(M) \neq 0$, proposition 2 gives

$$
\lambda_{M} \cong\left(-E_{8}\right) \oplus\left(\begin{array}{ll}
0 & 1 \\
1 & 0
\end{array}\right) \oplus\left(\begin{array}{cc}
1 & 0 \\
0 & -1
\end{array}\right) \cong 10(-1) \oplus 2(1),
$$

hence $M^{*} \underset{\text { TOP }}{\cong} 10\left(-C P^{2}\right) \# 2\left(C P^{2}\right)$ by the Freedman classification (see $[3])$.

We also recall that $\mathrm{C}$. Okonek (see [8]) has shown that all homotopy Enriques surfaces are homeomorphic to the Habegger manifold.

3.2) Let $M^{4}=S(\eta \oplus \eta \oplus \eta)$ be the sphcre bundle of $\eta \oplus \eta \oplus \eta$, where $\eta \longrightarrow R P^{2}$ is the canonical bundle over the real projective 2-space.

Then we have $\lambda_{M} \cong 0, w_{2}(M) \neq 0$ and $\Pi_{1}(M) \cong Z_{2}$, hence

$$
\lambda_{M^{*}} \cong\left(\begin{array}{cc}
1 & 0 \\
0 & -1
\end{array}\right)
$$

and $M^{*} \underset{\mathrm{TOP}}{\cong} C P^{2} \#\left(-C P^{2}\right) \underset{\mathrm{TOP}}{\cong} S^{2} \underset{\sim}{\simeq}$.

3.3) Let $M^{4}=S\left(\eta \oplus \epsilon^{2}\right)$ be the sphere bundle of $\eta \oplus \epsilon^{2}$, where $\epsilon^{2}=\epsilon^{1} \oplus \epsilon^{1} \longrightarrow R P^{2}$ is the 2-dimensional trivial bundle over $R P^{2}$.

Then wc have $\lambda_{M} \cong 0, w_{2}(M)=0$ and $\Pi_{1}(M) \cong Z_{2}$.

It is very easy to see that

$$
H^{2}\left(M ; Z_{2}\right) \underset{\text { iso }}{\stackrel{i^{*}}{\longrightarrow}} H^{2}\left(M_{0} ; Z_{2}\right) \underset{\text { isa }}{\stackrel{i^{\prime}-}{\longleftarrow}} H^{2}\left(M^{*} ; Z_{2}\right)
$$

where $M_{0}=M \backslash \psi\left(S^{1} \times \stackrel{\circ}{D}^{3}\right), \psi: S^{1} \times D^{3} \longrightarrow M$ represents the generator of $\Pi_{1}(M)$ and $i: M_{0} \rightarrow M, i^{\prime}: M_{0} \longrightarrow M^{*}$ are the natural inclusions.

Thus $w_{2}\left(M^{*}\right)=0$, hence $\lambda_{M^{*}} \cong\left(\begin{array}{ll}0 & 1 \\ 1 & 0\end{array}\right)$ is even and $M^{*} \underset{\text { TOP }}{\cong} S^{2} \times S^{2}$. 


\section{Proofs.}

Proof of proposition 2: For convenience we assume that $\Pi_{1}(M) \cong$ $Z_{m}, m>0$, with generator $[\alpha]=\left[\left.\psi\right|_{S^{1} \times 0}\right]$. For the general case, see remark 1 below.

We set $M_{0}=M \backslash \psi\left(S^{1} \times \stackrel{\circ}{D}^{3}\right)$ and consider the cobordism

$$
W=M \times I \cup_{\psi} D^{2} \times D^{3} \quad(I=[0,1])
$$

between $M$ and $M^{\prime}=M_{0} \cup D^{2} \times S^{2}$.

Obviously the pairs $(W, M),\left(W, M^{\prime}\right)$ are homology equivalent to $\left(D^{2} \times D^{3}, S^{1} \times D^{3}\right)$ and $\left(D^{2} \times D^{3}, D^{2} \times S^{2}\right)$ respectively.

We have the following diagram

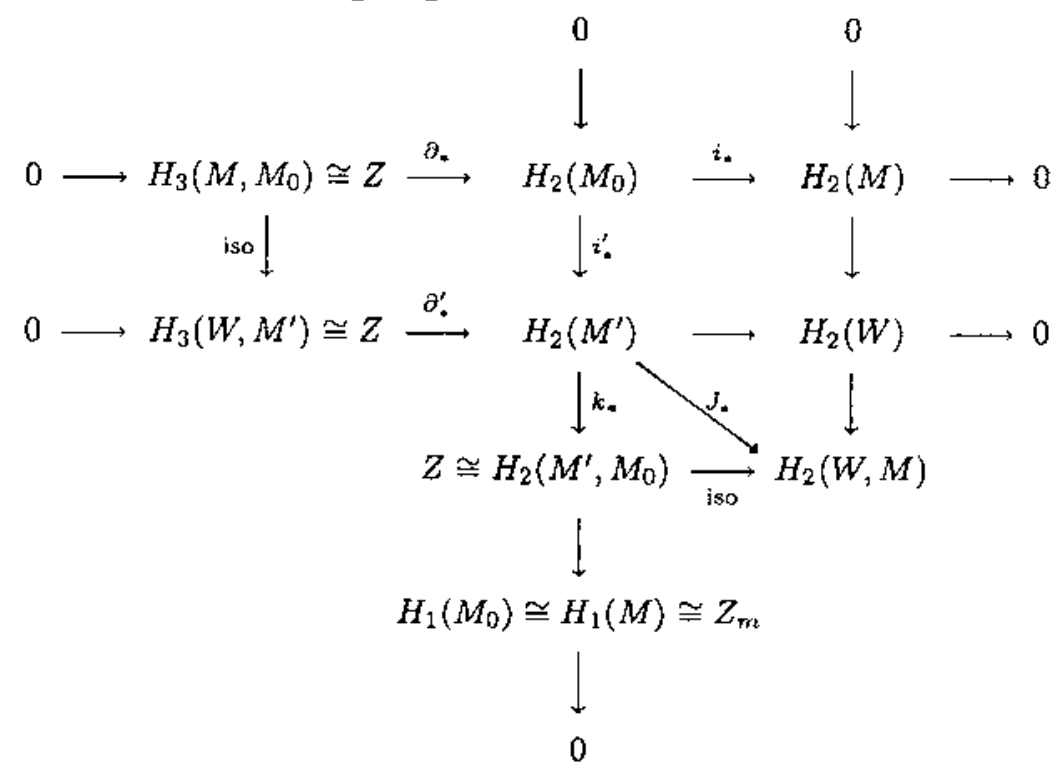

where $i, i^{\prime}, j, k$ are inclusions.

Obviously $\mathrm{H}_{2}\left(M^{\prime}\right)$ is a free group of rank $r k H_{2}(M)+2$ and $H_{2}\left(M_{0}\right)$ is free of rank $r k H_{2}(M)+1$ since it injects into $H_{2}\left(M^{\prime}\right)$.

Here we oftcn identify an clement of $H_{2}\left(M_{0}\right)$ with its image under $i_{*}^{\prime}$.

Now we have

$$
\lambda_{M}\left(i_{*}(u), i_{m}(v)\right)=\lambda_{M^{*}}\left(i_{*}^{\prime}(u), i_{*}^{\prime}(v)\right)
$$

for every $u, v \in H_{2}\left(M_{0}\right)$.

Let $e \in H_{2}\left(M_{0}\right)$ be a primitive elemert such that $i_{*}(e)$ generates the subgroup Tor $H_{2}(M) \cong Z_{m}$ and suppose that $f \in H_{2}\left(M^{\prime}\right)$ maps to the integer $m \in Z \cong H_{2}\left(M^{\prime}, M_{0}\right)$. Similarly $f$ is chosen to be primitive. Furthermorc, denote by $V$ the span of $\{e, f\}$ in $H_{2}\left(M^{\prime}\right)$. 
Lemma 6. With the above notation, we have

$$
\left.\lambda_{M^{\prime}}\right|_{V} \cong\left(\begin{array}{ll}
0 & 1 \\
1 & a
\end{array}\right)
$$

where $\lambda_{M^{\prime}}(f, f)=a \in Z$.

Proof: From the diagram, it follows that

$$
\lambda_{M^{\prime}}\left(\partial_{*}^{\prime}(x), y\right)=\lambda_{W}\left(x, j_{*}(y)\right)
$$

for every $x \in H_{3}\left(W, M^{\prime}\right)$ and $y \in H_{2}\left(M^{\prime}\right)$.

Note that

$$
\partial_{*}^{\prime}\left[D^{2} \times D^{3}, D^{2} \times S^{2}\right]=m i_{*}^{\prime}(e)=m e
$$

and

$$
j_{*}(f)=m\left[D^{2} \times D^{3}, S^{1} \times D^{3}\right]
$$

where [, ] denotes the fundamental class.

Thus relation (1) implies

$$
\begin{aligned}
\lambda_{M^{\prime}}(m e, f) & =\lambda_{M^{\prime}}\left(\partial_{*}^{\prime}\left[D^{2} \times D^{3}, D^{2} \times S^{2}\right], f\right) \\
& =\lambda_{W}\left(\left[D^{2} \times D^{3}, D^{2} \times S^{2}\right], j_{*}(f)\right) \\
& =m \lambda_{W}\left(\left[D^{2} \times D^{3}, D^{2} \times S^{2}\right],\left[D^{2} \times D^{3}, S^{1} \times D^{3}\right]\right)=m
\end{aligned}
$$

hence $\lambda_{M^{\prime}}(e, f)=1$ as required.

Furthermore, we have

$$
\begin{aligned}
m^{2} \lambda_{M^{\prime}}(e, e) & =\lambda_{M^{\prime}}(m e, m e) \\
& =\lambda_{M^{\prime}}\left(\partial_{*}^{\prime}\left[D^{2} \times D^{3}, D^{2} \times S^{2}\right], \partial_{*}^{\prime}\left[D^{2} \times D^{3}, D^{2} \times S^{2}\right]\right) \\
& =\lambda_{W}\left(\left[D^{2} \times D^{3}, D^{2} \times S^{2}\right], j_{*} \circ \partial_{*}^{\prime}\left[D^{2} \times D^{3}, D^{2} \times S^{2}\right]\right)=0
\end{aligned}
$$

since $j_{*} \circ \partial_{*}^{\prime}=0$ by the exactness. Thus $\lambda_{M}(e, e)=0$ and the proof of Lemma 6 is completed.

Lemma 7. Let $V^{\perp} \subset H_{2}\left(M^{\prime}\right)$ be the orthogonal complement of $V$. Then $V^{\perp} \subset H_{2}\left(M_{0}\right)$ and the restriction

$$
\left.i_{*}\right|_{V \perp}: V^{\perp} \longrightarrow F H_{2}(M)
$$

is an isomorphism.

Proof: To prove that $V^{\perp} \subset H_{2}\left(M_{0}\right)$, we have to show that for every $y \in H_{2}\left(M^{\prime}\right)$ with

$$
\lambda_{M^{\prime}}(y, e)=\lambda_{M^{\prime}}(y, f)=0,
$$


then $y \in H_{2}\left(M_{0}\right)$, i. e. $j_{*}(y)=0$.

Suppose, on the contrary, $j_{*}(y) \neq 0$, i. c. $j_{*}(y)=q\left[D^{2} \times D^{3}, S^{1} \times D^{3}\right]$ for some integer $q \neq 0$. Then we have

$$
\begin{aligned}
\lambda_{M^{\prime}}(m e, y) & =\lambda_{M^{\prime}}\left(\partial_{*}^{\prime}\left[D^{2} \times D^{3}, D^{2} \times S^{2}\right], y\right) \\
& =\lambda_{W}\left(\left[D^{2} \times D^{3}, D^{2} \times S^{2}\right], j_{*}(y)\right) \\
& =q \lambda_{W}\left(\left[D^{2} \times D^{3}, D^{2} \times S^{2}\right],\left[D^{2} \times D^{3}, S^{1} \times D^{3}\right]\right)=q \neq 0,
\end{aligned}
$$

hence $\lambda_{M^{\prime}}(e, y) \neq 0$, which is a contradiction.

To prove that $\left.i_{n}\right|_{V \perp}$ is mono, let $x \in V^{\perp}$ be an element such that i. $(x) \in \operatorname{Tor} H_{2}(M) \cong Z_{m}$.

Then we have $i_{*}(x)=h i_{*}(e)$ for some integer $h$, and so $i_{*}(x-h e)=0$.

By the exactness, it follows that

$$
\partial_{*}^{\prime}\left(h^{\prime}\left[D^{2} \times D^{3}, D^{2} \times S^{2}\right]\right)=i_{*}^{\prime}(x-h e),
$$

hence $m h^{\prime} e=x-h e, h, h^{\prime} \in Z$.

But we have (use (1))

(2)

$$
\begin{array}{r}
\lambda_{M^{\prime}}\left(\partial_{*}^{\prime}\left(h^{\prime}\left[D^{2} \times D^{3}, D^{2} \times S^{2}\right]\right), f\right)=\lambda_{W}\left(h^{\prime}\left[D^{2} \times D^{3}, D^{2} \times S^{2}\right], j_{*}(f)\right) \\
=\lambda_{W}\left(h^{\prime}\left[D^{2} \times D^{3}, D^{2} \times S^{2}\right], m\left[D^{2} \times D^{3}, S^{1} \times D^{3}\right]\right)=m h^{\prime}
\end{array}
$$

and

(3)

$$
\begin{aligned}
\lambda_{M^{\prime}}\left(\partial_{*}^{\prime}\left(h^{\prime}\left[D^{2} \times D^{3}, D^{2} \times S^{2}\right]\right), f\right) & =\lambda_{M^{\prime}}\left(i_{*}^{\prime}(x-h e), f\right) \\
& =\lambda_{M^{\prime}}(x-h e, f) \\
& =\lambda_{M^{\prime}}(x, f)-h \lambda_{M^{\prime}}(e, f)=-h .
\end{aligned}
$$

Comparing relations (2) and (3) gives $m h^{\prime}=-h$, hence $m h^{\prime} e=x-h e$ implies that $x=0$ as required.

To prove that $\left.i_{*}\right|_{\perp}$ is epi, let $z \in H_{2}(M)$ and let $u \in H_{2}\left(M_{0}\right)$ be an element such that $i_{m}(u)=z$.

We consider the element $u^{\prime}=u-\lambda_{M^{\prime}}(u, f) e \in H_{2}\left(M_{0}\right)$.

Then we have

$$
\begin{aligned}
\lambda_{M^{\prime}}\left(m e, u^{\prime}\right) & =\lambda_{M^{\prime}}\left(\partial_{*}^{\prime}\left[D^{2} \times D^{3}, D^{2} \times S^{2}\right], u^{\prime}\right) \\
& =\lambda_{W}\left(\left\{D^{2} \times D^{3}, D^{2} \times S^{2}\right], j_{*} \circ i_{*}^{\prime}\left(u^{\prime}\right)\right)=0
\end{aligned}
$$

since $j_{*} \circ i_{*}^{\prime}=0$; therefore $\lambda_{M^{\prime}}\left(u^{\prime}, e\right)=0$. 
Furthermore

$$
\begin{aligned}
\lambda_{M^{\prime}}\left(u^{\prime}, f\right) & =\lambda_{M^{\prime}}\left(u-\lambda_{M^{\prime}}(u, f) e, f\right) \\
& =\lambda_{M^{\prime}}(u, f)-\lambda_{M^{\prime}}(u, f)=0,
\end{aligned}
$$

i. e. $u^{\prime} \equiv i_{*}^{\prime}\left(u^{\prime}\right) \in V^{\perp}$.

Finally

$$
\begin{aligned}
i_{*}\left(u^{\prime}\right) & =i_{*}(u)-\lambda_{M}(u, f) i_{*}(c) \\
& =i_{*}(u)=z \bmod \operatorname{Tor} H_{2}(M)
\end{aligned}
$$

This completes the proof.

By Lemmas 6 and 7, we have the result

$$
\lambda_{M^{\prime}} \cong \lambda_{M} \oplus\left(\begin{array}{ll}
0 & 1 \\
1 & a
\end{array}\right) .
$$

Proof of Proposition 4:

Suppose now $w_{2}(M) \neq 0$. Because $\left(M, M_{0}\right)$ and $\left(M^{\prime}, M_{0}\right)$ are homology equivalent to $\left(S^{1} \times D^{3}, S^{1} \times S^{2}\right)$ and $\left(D^{2} \times S^{2}, S^{1} \times S^{2}\right)$ respectively, we have also the diagram

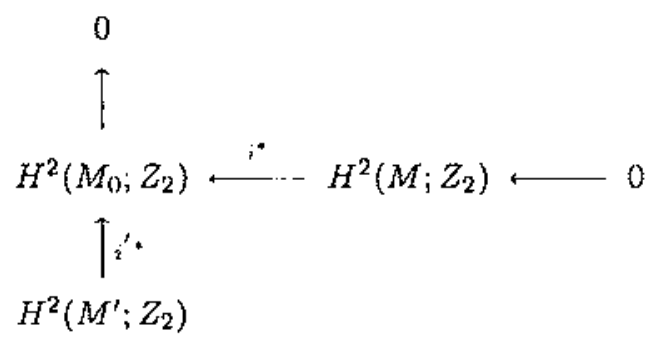

which implies

$$
i^{*}\left(w_{2}(M)\right)=w_{2}\left(M_{0}\right)=i^{*}\left(w_{2}\left(M^{\prime}\right)\right)
$$

Since $i^{*}$ is injective, relation (4) and $w_{2}(M) \neq 0$ give $w_{2}\left(M^{\prime}\right) \neq 0$, hence $\lambda_{M^{\prime}}$ is odd.

Remark 1. The proof of proposition 2 can be casily generalized to manifolds with arbitrary fundamental groups. Indeed, this follows from Lemma 8 below. 
Suppose now $M$ a closed connccted orientable (PL) 4-rnanifold with fundamental group $\Pi_{1}$.

Let

$$
\psi_{1}, \psi_{2}, \ldots, \psi_{p}: S^{1} \times D^{3} \longrightarrow M
$$

be disjoint embeddings which kill $\Pi_{1}$.

Setting

$$
M_{0}=M \backslash \bigcup_{j=1}^{p} \psi_{j}\left(S^{1} \times \stackrel{0}{D^{3}}\right)
$$

and

$$
M^{*}=M_{0} \cup \bigcup_{3=1}^{p}\left(D^{2} \times S^{2}\right)
$$

we have

\section{Lemma 8.}

(1) $H_{1}\left(M_{0}\right) \cong H_{1}(M), H_{3}\left(M_{0}\right) \cong \underset{p-1}{\oplus Z}$

(2) $\mathrm{H}_{2}\left(\mathrm{M}_{0}\right)$ is a direct summand of the free group $\mathrm{H}_{2}\left(\mathrm{M}^{*}\right)$

(3)

$$
\begin{aligned}
& 0 \longrightarrow H_{2}\left(M_{0}\right) \longrightarrow H_{2}\left(M^{*}\right) \longrightarrow H_{2}\left(M^{*}, M_{0}\right) \cong \underset{p}{\oplus} Z \longrightarrow \\
& \longrightarrow H_{1}\left(M_{0}\right) \cong H_{1}(M) \longrightarrow 0
\end{aligned}
$$

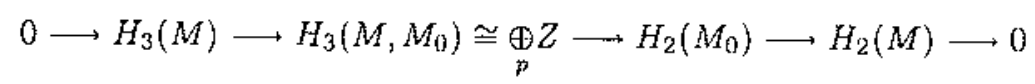

$$
\begin{aligned}
H_{2}(M) \cong H_{2}\left(M_{0}\right) \cong H_{2}\left(M^{*}\right) \Longleftrightarrow & \Longleftrightarrow H_{1}(M) \cong H_{3}(M) \cong H_{3}\left(M, M_{0}\right) \cong \underset{p}{\oplus} Z .
\end{aligned}
$$

The proof is straightforward.

Now we indicate how Lemma 8 yields Proposition 2 in the general case.

Suppose $\Pi_{1}(M)$ finitcly generated by elements of finites orders, hence 
$H_{1}(M)=Z_{m_{1}} \oplus \cdots \oplus Z_{m_{p}}$. Since $H_{3}(M) \simeq H^{1}(M) \simeq F H_{1}(M) \simeq 0$, by Lemma 8 we have the same diagram as at the beginning of section 4 with

$$
\begin{aligned}
H_{3}\left(M, M_{0}\right) & \simeq H_{3}\left(W, M^{*}\right) \simeq \oplus_{p} Z \\
H_{2}\left(M^{*}, M_{0}\right) & \simeq H_{2}(W, M) \simeq \oplus_{p} Z
\end{aligned}
$$

and

$$
H_{1}(M) \simeq H_{1}\left(M_{0}\right) \simeq Z_{m_{1}} \oplus \cdots \oplus Z_{m_{p}} .
$$

Obscrve that $\mathrm{H}_{2}\left(M^{*}\right)$ is a frec group of rank $r k H_{2}(M)+2 p$ and $H_{2}\left(M_{0}\right)$ is free of rank $\mathrm{r} k H_{2}(M)+p$.

Now we can choose primitive elements

$$
e_{1}, e_{2}, \ldots, e_{p} \in H_{2}\left(M_{0}\right) \text { and } f_{1}, f_{2}, \ldots, f_{p} \in H_{2}\left(M^{*}\right)
$$

such that $i_{*}\left(e_{h}\right)$ generates the subgroup $Z_{m h_{h}} \subset \operatorname{Tor} H_{2}(M)$ and $f_{h}$ maps to the integer $m_{h}$ which belongs to the corresponding $h^{5 l}$ factor of $H_{2}\left(M^{*}, M_{0}\right)$, for $h=1,2, \ldots, p$.

Now we apply the previons results by replacing $V$ with the span $V_{k}$ of $\left\{e_{h}, f_{h}\right\}$. As a consequence we also obtain

$$
\lambda_{M} *=\lambda_{M} \oplus\left(\begin{array}{cc}
0 & 1 \\
1 & a_{1}
\end{array}\right) \oplus \cdots \oplus\left(\begin{array}{cc}
0 & 1 \\
1 & a_{p}
\end{array}\right) .
$$

Remark 2. Let $M$ be a closed connected orientable spin 4-manifold with $\Pi_{1}(M)$ finitc.

Let $\psi: S^{1} \times D^{3} \longrightarrow M$ be an embedding which represents a generator $[\alpha] \in \Pi_{1}(M)$.

Then

$$
\lambda_{M} \cong \lambda_{M} \oplus\left(\begin{array}{ll}
0 & 1 \\
1 & a
\end{array}\right)
$$

by proposition 2 and $a$ defines a map

$$
\tilde{a}: \widetilde{\Pi_{1}(M)} \longrightarrow Z_{2}
$$

where $\widehat{\Pi_{1}(M)}$ is a certain extension of $\Pi_{1}(M)$ by $Z_{2}$ which takes care not only of $[\alpha]$ but also of its extension $\psi$ (for details sec $[10$, p. 44]). What type of invariant is $\tilde{a}$ ? : the examples show that $\tilde{a}$ is not trivial. 


\section{References}

1. A. Cavicchioli, F.Hegenbarth, Manifolds of type $C(p, q)$, Kobe Math. J. 7 (1990), 139-145.

2. S.K. DONALDSON, The orientation of Yang-Mills moduli spaces and 4-manifold topology, J. Diff. Geometry 26 (1987), 397-428.

3. M. FrEEDMAN, The topology of four-dimensional manifolds, $J$. Diff. Geometry 17 (1982), 357-453.

4. N. HABEGGER, Une varieté de dimension 4 avec forme d' intersection paire et signature -8 , Comment. Math. Helvetici 57 (1982), $22-24$.

5. R. Mandeldaum, Four-dimensional topology: an introduction, Bull. Amer. Math. Soc. 2 (1980), 1-159.

6. J. MrLnoR, A proccdure for killing homotopy groups of differentiable manifolds in, Proc. Symp. in Pure Math. 3, (Differential Geometry) Amer. Math. Soc., (1961), 39-55.

7. J. Milnor, D. Husemolier, "Symmetric bilinear forms," SpringerVerlag Ed., Berlin-Heidelberg-New York, 1973.

8. C. ОKОNEK, Fake Enriques surfaces, Topology 27 (1988), 415-427.

9. O.T. O' MEakA, "Introduction to quadratic forms," Springer-Verlag Ed., Berlin-Heidelberg-New York, 1963.

10. C.T.C. WALL, "Surgery on compact manifolds," Academic Press, London-New York, 1970.

Alberto Cavicchioli:

Dipartimento di Matematica

Universitá di Modena

Via Campi 213/B

41100 Modena

ITALY
Friedrich Hegenbarth:

Dipartimento di Matematica

Universitá di Tor Vergata

Via Fontanile di Caracaricola 00133 Roma

ITALY

Rebut el 12 de Desembre de 1990 